\title{
Design De Jogos Digitais Terapêuticos Por Usuários Finais Com Transtornos Mentais Mediado Por Agentes Inteligentes
}

\author{
Paula Maia de Souza \\ Universidade Federal de São Carlos - UFSCar \\ São Carlos, São Paulo, Brazil \\ paula.souza@ufscar.br
}

\author{
Vânia Paula de Almeida Neris \\ Universidade Federal de São Carlos - UFSCar \\ São Carlos, São Paulo, Brazil \\ vania@dc.ufscar.br
}

\begin{abstract}
RESUMO
O Laboratório de Interação Flexível e Sustentável está construindo uma ferramenta que visa permitir o desenvolvimento de jogos por usuários finais. A ferramenta não foi desenvolvida com finalidade terapêutica, porém foi aplicada com pacientes do Centro de Atenção Psicossocial Álcool e Drogas de São Carlos - SP. Essa aplicação foi tutorada por agentes humanos (profissionais da computação e terapeutas do CAPS-AD), que observaram potencial pesquisa no sentido de analisar o seu reflexo terapêutico nos pacientes. Dentro desse contexto, o objetivo deste trabalho consiste em investigar o design de jogos digitais terapêuticos por usuários finais com transtornos mentais. Após os resultados dessa investigação, buscar-se-a propor uma solução para apoiar os usuários finais com transtornos mentais a fazerem design e programarem jogos digitais terapêuticos, sem a necessidade da presença física de profissionais da computação. O trabalho também busca investigar se o ato de construir jogos pode de fato auxiliar no tratamento dos pacientes. Tendo como foco os objetivos propostos, buscas na literatura estão sendo realizadas para identificar o estado da arte relativo ao tema. Paralelamente, uma pesquisa utilizando o método de pesquisa-ação será aplicada, visando identificar as dificuldades dos usuários finais (pacientes) em construir o design e implementar jogos digitais terapêuticos. Após análise qualitativa dos dados levantados, uma solução será proposta e a mesma será avaliada por meio de um estudo de caso.
\end{abstract}

\section{PALAVRAS-CHAVE}

Design, jogos digitais terapêuticos, agentes inteligentes, desenvolvimento por usuário final.

\section{INTRODUÇÃO}

A aplicação dos saberes em computação visando apoiar outras áreas de conhecimento é uma realidade no cenário mundial [4]. Sistemas são desenvolvidos buscando melhorar a vida das pessoas, individual e coletivamente.

A criação de sistemas computacionais para auxiliar a área da saúde está em crescente expansão [17]. Tem-se desenvolvido desde programas aplicativos que apoiam os profissionais da saúde em

Permission to reproduce or distribute, in whole or in part, material extracted from this work, verbatim, adapted or remixed, as well as the creation or production from the content of such work, is granted without fee for non-commercial use, provided that the original work is properly credited.

IHC 2019 - WORKSHOP DE TESES E DISSERTAÇÕES, Outubro 2125, 2019, Vitória, Brasil. In Anais Estendidos do XVIII Simpósio Brasileiro sobre Fatores Humanos em Sistemas Computacionais. Porto Alegre: SBC.

(c) 2019 by the author(s), in accordance with the terms of the Creative Commons Attribution-NonCommercial 4.0 International Public License (CC BY-NC 4.0). seus consultórios (e.g. planilhas eletrônicas, softwares para controle financeiro e de pacientes, etc.), até jogos digitais criados para auxiliar diretamente no tratamento de pacientes.

No contexto de jogos digitais, o Laboratório de Interação Flexível e Sustentável (LIFeS) do Departamento de Computação (DC) da Universidade Federal de São Carlos (UFSCar), vem desenvolvendo uma ferramenta que visa apoiar a criação de jogos por e para todos. A ferramenta, nomeada Lepi, está pautada em duas frentes principais: a criação de jogos digitais e o uso desses jogos criados [5].

A Lepi foi aplicada com pacientes do Centro de Atenção Psicossocial Álcool e Drogas (CAPS-AD) de São Carlos - SP. Nas atividades no CAPS-AD os pesquisadores observaram que a atividade de criar jogos propiciou certo efeito terapêutico nos pacientes [5].

As atividades porém foram restritas a uma hora a cada quinze dias. Essa restrição se deu pela inviabilidade dos pesquisadores da computação estarem presentes no CAPS-AD por mais tempo. E, apesar da Lepi apoiar a construção de jogos por e para todos, os pacientes (usuários finais) tiveram a necessidade de apoio dos profissionais da computação para que conseguissem criar os jogos.

Nesse contexto, os pesquisadores se sentiram motivados a prover um meio para apoiar o desenvolvimento de jogos digitais por usuários finais sem a presença física de profissionais da computação. E pesquisar sobre como o desenvolvimento de jogos digitais terapêuticos por usuários finais pode auxiliar na terapia dos mesmos.

O LIFeS já vem pesquisando sobre design de jogos digitais terapêuticos, sendo que uma abordagem foi formalizada para esta categoria de jogos. A SemTh $[14,15]$ é uma abordagem participativa que apoia profissionais multidisciplinares no design de jogos digitais terapêuticos.

Um mapeamento sistemático inicial foi realizado com o intuito de levantar o estado da arte em relação a sistemas de tutoria inteligente para o ensino de programação de jogos digitais terapêuticos. O mapeamento apontou uma carência de trabalhos relacionados na literatura e, a partir dessa carência, definiu-se a problemática sobre como tutorar o design e programação de jogos digitais terapêuticos por usuários finais, sem a presença física de profissionais da computação.

Considerando o que foi exposto, o objetivo deste trabalho consiste em investigar o design de jogos digitais terapêuticos por usuários finais com transtornos mentais. Analisando os potenciais efeitos terapêuticos e buscando um meio para apoiar (tutorar) esses usuários de forma não presencial, visando uma solução escalável.

Como objetivos específicos destacam-se:

- Levantar o estado da arte sobre design de jogos digitais terapêuticos e end-user development; 
- Investigar os desafios encontrados por usuários finais com transtornos mentais em fazer design e programar jogos digitais terapêuticos;

- Criar uma solução que apoie usuários finais com transtornos mentais a fazerem design e programar jogos digitais terapêuticos, sem a presença física de profissionais da computação.

\section{FUNDAMENTAÇÃO TEÓRICA}

Nesta seção apresenta-se a base teórica que fundamenta o projeto de pesquisa.

\subsection{Design de Jogos Digitais Terapêuticos}

Os jogos digitais podem ser definidos como ambientes atraentes e interativos que capturam a atenção do jogador ao oferecer desafios que exigem níveis crescentes de destreza e habilidades [1]. Eles também oferecem formas de interação dinâmicas impossíveis de se observar em ambientes não digitais e maior rigidez das regras, já que nos jogos não digitais sempre existe um espaço para negociação entre os jogadores [9].

Com a expansão do uso de tecnologias, os jogos digitais evoluíram para além do entretenimento, surgindo o conceito de Serius Games (SG). Os SG são aplicações computacionais interativas que proporcionam metas desafiadoras de uma forma divertida. Os serius games podem atuar na área da educação, formação profissional, saúde, entre outras [7] . Os jogos digitais terapêuticos se enquadram na categoria de SG voltados para a saúde.

$\mathrm{O}$ termo terapia consiste em qualquer tratamento pretendido $\mathrm{e}$ esperado para aliviar doenças ou desordens. Qualquer técnica de recuperação que possa ser médica, psiquiátrica ou psicológica é considerada uma técnica terapêutica [11]. Com base nessa definição de terapia, pode-se considerar os jogos digitais terapêuticos como jogos que produzem efeito terapêutico direto, esperado e pretendido sobre os pacientes que o jogam. Esse efeito terapêutico pode aliviar, melhorar ou curar a condição específica dos pacientes [10] .

O design do jogo é o processo pelo qual um designer de jogos cria um jogo. Para um jogo terapêutico, o designer do jogo precisa definir uma jogabilidade a partir da qual emergem sessões de jogo significativas e motivadoras [10]. Com base nos estudos sobre jogos digitais, acredita-se que o design de jogos digitais terapêuticos se difere dos demais pela necessidade de participação de especialistas no domínio da terapia a ser atendida [10].

\subsection{SemTh}

A SemTh é uma abordagem para o design de jogos digitais terapêuticos que apoia a participação de diferentes profissionais durante todo o processo de design. Ela possui etapas iterativas, que apoiam a participação e colaboração de profissionais da computação, profissionais da saúde e pacientes no processo de construção de jogos orientados ao contexto da patologia a ser atendida [14,15]. A abordagem é composta por quatro etapas, sendo elas: 1) Clarificação do Problema de Design, 2) Modelagem da Interação, 3) Materialização do Design e 4) Avaliação.

A Clarificação do Problema de Design visa entender o domínio no qual o jogo será aplicado e, como resultado desta etapa, propor um estilo para esse jogo [14]. Como resultado da etapa de Clarificação do Problema de Design deve-se especificar a mecânica, a estética, a narrativa e a tecnologia que serão aplicadas ao jogo a ser desenvolvido.

A Modelagem da Interação consiste em modelar as interações para o jogo. Pelo fato dos jogos terapêuticos estarem em um contexto multidisciplinar, a SemTh sugere o uso da linguagem de modelagem de domínio específico para aplicações terapêuticas [6, 14]. A linguagem foi criada com a intenção de facilitar a comunicação no contexto multidisciplinar. Como resultado desta etapa de modelagem tem-se a especificação das cenas (interações) do jogo [14].

A Materialização do Design é a terceira etapa da SemTh e consiste em criar protótipos e realizar a implementação do jogo. Os protótipos podem ser desde protótipos em papel até versões iniciais codificadas do jogo. Como resultado desta etapa, tem-se uma versão executável do jogo proposto [14].

A etapa de Avaliação consiste em avaliar o jogo desenvolvido ou os protótipos do jogo. Essa avaliação deve ser realizada pela equipe executora do projeto e pelos usuários (pacientes). Caso necessário, deve-se voltar à etapas anteriores para correções e/ou adição de novos requisitos, fechando o fluxo proposto pela abordagem [14].

\subsection{End-User Development}

End-User Development (EUD) é um campo de pesquisa que estuda técnicas e métodos para capacitar os usuários finais a modificarem e criarem artefatos digitais [2]. Visando permitir que usuários finais criem novas funcionalidades ou modifiquem as existentes em sistemas computacionais [8]. Seu objetivo principal é empoderar os usuários, ao permitir que eles mesmos desenvolvam ou adaptem sistemas para satisfazer às suas necessidades [8].

EUD é um conjunto de práticas, métodos, técnicas e ferramentas que propiciam aos usuários finais a oportunidade de criarem, modificarem ou estenderem um software [8]. Alguns principais conceitos e práticas para abordagens de EUD são: i) configurability, que caracteriza-se pela capacidade de configuração e modificação dos sistemas; ii) tailorability, que aponta que a modificação de um sistema deve considerar seu contexto de uso; iii) gentle slope, aborda que é importante oferecer ao usuário uma curva de aprendizagem sutil; iv) direct manipulation, procura proporcionar que as alterações em um artefato sejam realizadas no próprio artefato (manipulação direta); v) visual programming, está relacionado à necessidade de promover a manipulação de componentes da linguagem de programação de maneira visual; e vi) natural programming, tem por objetivo oferecer um sistema de programação que funcione de forma natural, ou seja, da forma que as pessoas esperam e compreendam $[8,19]$.

\subsection{Design Participativo}

O Design Participativo (DP) surgiu na Escandinávia, dentro de um contexto voltado para a participação de funcionários no processo de desenvolvimento de sistemas [3]. O DP agrega um conjunto de estudos, teorias e práticas relacionadas com usuários e profissionais de desenvolvimento de software, hardware ou qualquer atividade relacionada ao computador [12]. O DP apresenta uma abordagem valiosa a ambientes inclusivos de design, pois oferece técnicas que apoiam a participação direta de usuários em diferentes fases de design [13]. 
Dentre os benefícios do DP destacam-se a melhoria na aprendizagem, da compreensão do sistema e maior participação das partes interessadas [12]. Para a coleta, análise e projeto podem ser utilizados cenários para descrever situações cotidianas dos envolvidos. Pode-se ainda elaborar protótipos em papel ou informatizados [3].

No domínio de aplicações terapêuticas há a necessidade de participação de diferentes profissionais no processo de criação e desenvolvimento. O DP foi adotado como referencial por trazer princípios que proporcionam melhores condições de comunicação entre as diferentes partes interessadas.

\section{PROPOSTA}

A programação por usuários finais é um conceito já fundamentado na literatura. A participação desses usuários finais, bem como de diferentes partes interessadas, também já é fortemente apoiada pela literatura, principalmente pelas técnicas de design participativo. Porém, os jogos digitais terapêuticos possuem particularidades e demandam atenção especial na sua construção [14].

A proposta deste trabalho consiste em investigar o design de jogos digitais terapêuticos por usuários finais com transtornos mentais. Nessa investigação buscar-se-a identificar quais as dificuldades desses usuários em criar esses jogos e qual o potencial efeito terapêutico exercido sobre os usuários. Os dados dessa investigação serão analisados de forma qualitativa e com base neles será proposta uma solução para apoiar esses usuários.

A intenção dos pesquisadores é tornar essa solução escalável, ou seja, que possa ser utilizada por vários usuários finais e apoiar no tratamento de transtornos mentais. Sendo assim, pretende-se criar uma solução inteligente, que possa auxiliar os usuários finais no processo de design sem a presença física de profissionais da computação.

Para isso, acredita-se que utilizando técnicas de inteligência artificial, por meio de agentes inteligentes e Sistemas Tutores Inteligentes (STI), será possível mediar esse design. Os agentes inteligentes são representações de humanos em um ambiente virtual [16]. Assim, acredita-se ser possível criar um 'designer virtual inteligente' para apoiar esses usuários finais com transtornos mentais a fazerem design de jogos digitais terapêuticos.

\section{METODOLOGIA}

Para o desenvolvimento deste projeto serão realizadas pesquisas bibliográficas, na busca de se conhecer a literatura dentro do contexto. Os métodos de Mapeamento Sistemático e Revisão Sistemática serão utilizados para delimitar o estado da arte em relação ao EUD, design de jogos digitais terapêuticos por usuários finais com transtornos mentais e STI.

Um mapeamento sistemático preliminar foi realizado na intenção de obter uma visão geral sobre o estado da arte relacionado ao uso de STI para o ensino de programação de jogos digitais terapêuticos. As buscas foram realizadas na ACM Digiital Library, IEEE Xplore e Google Scholar. As questões de pesquisa buscavam identificar o que o tutor inteligente ensina, como ele funciona e qual o público alvo.

As buscas revelaram uma carência na literatura de trabalhos relacionados ao tema. Foram encontrados trabalhos apenas sobre STI para ensino de programação em geral, mas não específicos sobre jogos digitais terapêuticos. Nos próximos passos da pesquisa será realizada uma revisão sistemática mais detalhada sobre o tema, envolvendo também EUD e conceitos como end-user design e open design.

Em paralelo às pesquisas na literatura, o método de pesquisaação [18] será utilizado. Na busca de investigar o design de jogos digitais terapêuticos por usuários finais com transtornos mentais, a abordagem SemTh e a ferramenta Lepi serão aplicadas com um grupo de usuários finais com transtornos mentais e os profissionais da saúde que os acompanham. O objetivo é coletar dados sobre quais as dificuldades que os usuários finais terão em fazer esse design e em que eles precisarão ser apoiados por profissionais da computação. Os dados serão coletados dados por meio de observação, imagens, áudios, vídeos, questionários e entrevistas e, posteriormente, serão analisados por meio de análise qualitativa.

Após a análise desses dados será proposta uma solução inteligente que possa apoiá-los nas dificuldades que tiveram, sem que haja a necessidade de presença física de profissionais da computação. Técnicas de Inteligência Artificial, como agentes inteligentes [16], tendem a serem utilizadas para proposta da solução.

A solução será avaliada por meio de um estudo de caso. Disponibilizando a solução para usuários finais com transtornos mentais buscar-se-a verificar se os mesmos conseguiram fazer design e desenvolver jogos digitais terapêuticos com mediação de um designer não humano. No estudo de caso os dados também serão coletados por meio de observação, imagens, áudios, vídeos, questionários e entrevistas e serão analisados por meio de análise qualitativa.

\section{CONTRIBUIÇÕES ESPERADAS}

Com a realização deste projeto de pesquisa espera-se preencher uma lacuna na literatura sobre o design de jogos digitais terapêuticos por usuários finais com transtornos mentais. Espera-se que este trabalho traga contribuições para a área de Interação Humano Computador (IHC), formalizando um meio para que usuários finais utilizem dos conhecimentos de design para a construção de jogos digitais terapêuticos. E espera-se também que este trabalho traga contribuições para a área da saúde, oferecendo uma solução que possa apoiar os profissionais em atividades terapêuticas com pacientes com transtornos mentais.

\section{AGRADECIMENTOS}

O presente trabalho está sendo realizado com apoio da Coordenação de Aperfeiçoamento de Pessoal de Nível Superior - Brasil (CAPES) - Código de Financiamento 001.

\section{REFERÊNCIAS}

[1] Nathan Balasubramanian, Brent Wilson, and Krzysztof Cios. 2006. Innovative Methods of Teaching Science and Engineering in Secondary Schools. (2006). http://source.ucdenver.edu/ilt_publications/22

[2] B. R. Barricelli, F. Cassano, D. Fogli, and A. Piccinno. 2018. End-user development, end-user programming and end-user software engineering: A systematic mapping study. Journal of Systems and Software 149 (March 2018), 101-137. https: //doi.org/10.1016/j.jss.2018.11.041

[3] Liriane Soares de Araújo Camargo and Alex Jose Fazani. 2014. Explorando o Design Participativo como Prática de Desenvolvimento de Sistemas de Informação. 5, 1 (2014), 138-150. https://doi.org/10.11606/issn.2178-2075.v5i1p138-150

[4] L. Carro and F. R. Wagner. 2016. Desafios para a computação pervasiva no futuro cenário tecnológico. In Grandes desafios computação no Brasil 2006-2016. Campinas, SP. http://www.ic.unicamp.br/ cmbm/desafios_SBC/Carro_Wagner. pdf 
[5] Franco Eusébio Garcia. 2019. An Inclusive End-User Development Framework for Tailorable Games. Tese Doutorado em Ciência da Computação, Departamento de Computação, Universidade Federal de São Carlos - UFSCar, São Carlos, SP. 321 pages.

[6] Franco Eusébio Garcia, Kamila Rios da Hora Rodrigues, and Vânia Paula de Almeida Neris. 2016. An Interaction Modeling Language for Therapeutic Applications. In Proceedings of the 15th Brazilian Symposium on Human Factors in Computer Systems (IHC '16). ACM, 32:1-32:10. https://doi.org/10.1145/3033701.3033733

[7] D. O. Lemes. 2014. Serious games - jogos e educação. http://www.abrelivros.org br/home/index.php/bienal-2014/resumos-e-fotos/5647-primeiro-resumo

[8] H. Lieberman, F. Paternó, M. Klann, and V. Wulf. 2006. End-User Development: An Emerging Paradigm. In End User Development, Henry Lieberman, Fabio Paternò, and Volker Wulf (Eds.). Springer Netherlands, Dordrecht, 1-8. https //doi.org/10.1007/1-4020-5386-X_1

[9] F. Lucchese and B. Ribeiro. 2009. Conceituação de jogos digitais. (2009). http: //www.dca.fee.unicamp.br/ martino/disciplinas/ia369/trabalhos/t1g3.pdf

[10] S. Mader, G. Levieux, and S. Natkin. 2016. A Game Design Method for Therapeutic Games. In 2016 8th International Conference on Games and Virtual Worlds for Serious Applications (VS-GAMES) (2016-09). 1-8. https://doi.org/10.1109/VSGAMES.2016.7590333

[11] T. MCGraw, K. Burdette, and K. Chadwick. 2005. The effects of a consumeroriented multimedia game on the reading disorders of children with adhd. (2005)

[12] Michael J. Muller. 2002. The Human-computer Interaction Handbook. L. Erlbaum Associates Inc., Hillsdale, NJ, USA, 1051-1068. http://dl.acm.org/citation.cfm? id $=772072.772138$
[13] Michael J. Muller, Jean Hallewell Haslwanter, and Tom Dayton. 1997. Participatory Practices in the Software Lifecycle. In Handbook of Human-Computer Interaction (Second Edition), Marting G. Helander, Thomas K. Landauer, and Prasad V. Prabhu (Eds.). North-Holland, Amsterdam, 255-297.

[14] P. M. Souza. 2018. Abordagem para o Design de fogos Digitais Terapêuticos. 115p. Dissertação de mestrado. Universidade Federal de São Carlos. São Carlos, SP.

[15] P. M. d. Souza, K. R. d. H. Rodrigues, F. E. Garcia, and V. P. d. A. Neris. 2018. Towards a Semiotic-Based Approach to the Design of Therapeutic Digital Games. In Digitalisation, Innovation, and Transformation (IFIP Advances in Information and Communication Technology), Kecheng Liu, Keiichi Nakata, Weizi Li, and Cecilia Baranauskas (Eds.). Springer International Publishing, 53-62.

[16] Scott Stiefel. 2018. 'The Chatbot Will See You Now': Mental Health Confidentiality Concerns in Software Therapy. SSRN Scholarly Paper ID 3166640. Social Science Research Network, Rochester, NY. https://papers.ssrn.com/abstract $=3166640$

[17] C. M. d. S. Tibes, J. D. Dias, and S. H. Zem-Mascarenhas. 2014. Aplicativos móveis desenvolvidos para a área da saúde no Brasil: revisão integrativa da literatura. Revista Mineira de Enfermagem (2014), 471-486. https://doi.org/10.5935/14152762.20140035

[18] David Tripp. 2005. Action research: a methodological introduction. Educação e Pesquisa 31, 3 (Dec. 2005), 443-466. https://doi.org/10.1590/S151797022005000300009

[19] M. Won, O. Stiemerling, and V Wulf. 2006. Component-Based Approaches to Tailorable Systems. In End User Development, F.; WULF V. LIEBERMAN, H.; PATERNÒ (Ed.). Springer Netherlands, p. 115-141. 